\title{
Beclin-1 is a Promising Prognostic Biomarker in a Specific Esophageal Squamous Cell Carcinoma Population
}

\author{
Hailei Du ${ }^{1}$, Fangxiu Luo ${ }^{2}$, Minmin Shi ${ }^{3}$, Jiaming Che ${ }^{1}$, Lianggang $Z h u^{1}$, Hecheng $L_{i}{ }^{1}$ and \\ Junbiao Hang ${ }^{1 *}$ \\ ${ }^{1}$ Department of Thoracic Surgery, Ruijin Hospital, Shanghai Jiao Tong University School of Medicine, Shanghai, China, \\ ${ }^{2}$ Department of Pathology, Ruijin Hospital North, Shanghai Jiao Tong University School of Medicine, Shanghai, China, ${ }^{3}$ Institute of \\ Digestive Surgery, Shanghai, China
}

OPEN ACCESS

Edited by:

József Tímár.

Semmelweis University, Hungary

*Correspondence:

Junbiao Hang

drhangjb@hotmail.com

Received: 14 August 2020 Accepted: 12 February 2021

Published: 09 April 2021

Citation:

Du H, Luo F, Shi M, Che J, Zhu L, Li H and Hang $J$ (2021) Beclin-1 is a Promising Prognostic Biomarker in a Specific Esophageal Squamous Cell

Carcinoma Population.

Pathol. Oncol. Res. 27:594724.

doi: 10.3389/pore.2021.594724
The effects of autophagy and apoptosis in the prognostic assessment and treatment of Esophageal squamous cell carcinoma (ESCC) remain to be elucidated. Here, we conducted a retrospective study on the histopathology of ESCC, investigated the expression of Beclin-1 and Bcl-2 proteins (both autophagy- and apoptosis-related) in esophageal cancer tissue, and analyzed the significance of these proteins for the prognosis of ESCC. In the present study, the expression level of Beclin-1 in ESCC was significantly lower than that in adjacent tissues $(p<0.01)$, whereas the expression level of Bcl-2 showed the opposite pattern $(p<0.01)$. Furthermore, low expression of Beclin-1 was associated with more advanced ESCC stages and lymph node metastasis. However, high expression of Bcl-2 was associated with more advanced ESCC stages, deeper tumor invasion, and lymph node metastasis. Moreover, the relationship between Bcl-2 expression and OS was not significant $(p>0.05)$, whereas Beclin-1 expression was significantly associated with OS $(p<0.05)$. Subgroup analysis showed that Beclin-1 expression was significantly associated with OS in the high-Bcl-2-expression group but not in the low-Bcl-2-expression group. Importantly, Beclin-1 upregulation or downregulation significantly upregulated or downregulated invasion, respectively, in EC9706 cells in combination with high expression but not low expression of Bcl-2. These findings reveal that differences in autophagy and apoptotic states and their activities may promote malignant tumor differentiation, which could lead to a more aggressive esophageal squamous cell phenotype and a worse survival prognosis. Here, Beclin-1 was shown to be a promising prognostic biomarker and therapeutic target for patients with ESCC in the high-Bcl-2-expression population.

Keywords: esophageal squamous cell carcinoma (ESCC), autophagy, apoptosis, beclin-1, Prognosis

\section{INTRODUCTION}

Esophageal cancer is one of the most invasive cancers, and consequently ranks as the sixth most common cause of cancer death worldwide [1]. Esophageal squamous cell carcinoma (ESCC), which is the main histological subtype of esophageal cancer with almost $90 \%$ of esophageal cancer being ESCC [2], has high morbidity and mortality. Despite improved diagnostic and treatment strategies such as endoscopic resection, targeted therapy, and immunotherapy, the overall survival (OS) of 
ESCC patients remains poor. For example, the 5-years survival rate for patients with advanced esophageal cancer is $<20 \%$ [3]. Therefore, to improve the prognosis of patients with ESCC, it is important to understand how to improve early diagnosis, identify new prognostic markers, and find new therapeutic targets.

Autophagy is a conservative pathway that exists in eukaryotic cells [4]. It degrades and removes damaged organelle components in cells to maintain the homeostasis of cells and provides nutritional support for cell survival [5]. Previous studies have found that abnormal autophagy is associated with a variety of human diseases such as Alzheimer's disease, cardiovascular disease, and several types of cancer including ESCC [6-8]. As the first autophagy-specific gene identified, Beclin-1 plays an important role in the regulation of autophagy. Changes in Beclin1 expression levels have been described in a variety of cancers [9-11]. However, the association between Beclin-1 and the pathogenesis of tumors is yet to be studied in detail, and correlation results between the two remain controversial.

B-cell lymphoma-2 (Bcl-2) protein is a regulator of both autophagy and apoptosis. There are two sources of Bcl-2: Bcl2 in the ER (ER-Bcl-2) and mitochondrial Bcl-2 (mito-Bcl-2). Research indicates that these Bcl-2 proteins from different sources perform different roles in the regulation of autophagy and apoptosis [12]. Therefore, investigating how different Bcl-2 expression levels shift from regulating autophagy to apoptosis is important. In addition, the relationship between Beclin-1 and Bcl-2 in ESCC has not yet been fully elucidated.

Therefore, the central purpose of our study was to analyze the relationships between both Beclin-1 and Bcl-2 and the clinicopathological characteristics of patients with ESCC. Furthermore, we investigated the effect of Beclin-1 on the prognosis of ESCC patients with different expression levels of Bcl-2.

\section{METHODS AND MATERIALS}

\section{Patients and Tissue Samples}

Patients who underwent surgical resection of esophageal cancer at the Shanghai Ruijin hospital from July 2013 to July 2014 were selected for this study. All patients were diagnosed with ESCC after surgery and patients who received neoadjuvant therapy or showed postoperative complications were excluded: 102 patients with ESCC were ultimately included. All tissue specimens were acquired from pathological paraffin sections. The study group included 65 males and 37 females (age: 45-75 years; median age: 63.5 years). Patients were classified into stages I, II, III, and IV according to the eighth edition of the TNM staging of esophageal cancer. The follow-up observation endpoint was July 2019. This study was approved by the Clinical Ethics Committee of Ruijin hospital.

\section{Immunohistochemistry (IHC) Staining and Evaluation}

Formalin-fixed and paraffin-embedded 5- $\mu$ m ESCC tissues were deparaffinized in xylene, and then sequentially rehydrated in a range of alcohol concentrations before being washed three times with PBS. IHC staining was performed using primary anti-Beclin1 (1:200 dilution; Santa Cruz, SC-11427) and anti-Bcl-2 (1:100 dilution; Abcam, ab32124) incubated at $4^{\circ} \mathrm{C}$ overnight. Slices were then incubated for $15 \mathrm{~min}$ with a biotin-labeled secondary antibody before being washed with PBS three times. Hematoxylin staining was performed for $2 \mathrm{~min}$ to counterstain. Two different pathologists judged the intensity of the immunostaining in a blinded manner. Staining was scored separately using two methods as follows. Scoring standard A: $\leq 10 \%$ positive cells $=1$ point; $11 \%-50 \%$ positive cells $=2$ points; $51-75 \%$ positive cells $=3$ points; $>75 \%$ positive cells $=4$ points. Staining intensity score $\mathrm{B}$ : $0=$ negative; 1 = light yellow; 2 = brownish-yellow; 3 = brownish. The terminal score was determined as $\mathrm{A} \times \mathrm{B}$. Where this score was $\leq 3$, the subject was classified into the low expression group; where this score was $>3$, the subject was classified into high expression group.

\section{Small Interfering RNA (siRNA)}

Bcl-2 siRNA (cat. no. 29214, Santa Cruz) and Beclin-1 siRNA (cat. no. 137198, Invitrogen) were transfected using the Lipofectamine RNAiMAX Transfection reagent (cat. no. 13778-075, Invitrogen) according to the recommended protocol. After $24 \mathrm{~h}, \mathrm{Bcl}-2$ and Beclin-1 western blotting was performed to detect the respective expression of each protein. Control Bcl-2 siRNA (cat. no. 37007, Santa Cruz) and control Beclin-1 siRNA (cat. no. 12935400, Invitrogen) were used as controls, respectively.

\section{Construction of Overexpression Vectors}

Recombinant pcDNA3.1-Bcl-2 plasmid and pcDNA3.1-Beclin-1 plasmid (provided by Dr. Qin Ye and Ruyuan Zhang, respectively, Shanghai Institute of Digestive Surgery, Shanghai Jiao Tong University School of Medicine) were transfected into human esophageal carcinoma EC9706 cells by lipofectamine 2000. Bcl-2 and Beclin-1 western blotting was then performed as previously described protocols [13].

\section{Isolation of Proteins and Western Blot Analysis}

The cells were lyzed in lysis buffer and then high-speed centrifuged at $12,000 \mathrm{rpm}$, for $10 \mathrm{~min}$. A BCA assay was used to quantify protein concentrations. The supernatant was separated on $12.5 \%$ SDS-PAGE gel and transferred to a polyvinylidene membrane. After transfer, the membrane was blocked with $5 \%$ non-fat milk and incubated with specific primary antibodies as follows. Incubation with Beclin-1 (cat. no. 48341, Santa Cruz) and Bcl-2 (cat. no. 509, Santa Cruz) was conducted overnight at $4^{\circ} \mathrm{C}$. After incubation with a secondary antibody, the specific proteins were detected by enhanced chemical luminescence.

\section{Transwell Migration Assay}

An $8-\mu \mathrm{m}$ inserted Transwell chamber (Corning Incorporated, NY, United States) was used to examine cell migration with or without matrigel. First, $6 \times 104$ cells in $200 \mu \mathrm{L}$ of DMEM without 
TABLE 1 | Expression of Beclin-1 and Bcl-2 in ESCC tissues and adjacent tissues.

\begin{tabular}{|c|c|c|c|c|c|c|}
\hline \multirow[t]{2}{*}{ Organization type } & \multicolumn{2}{|c|}{ Beclin-1 expression } & \multirow[t]{2}{*}{$p$} & \multicolumn{2}{|c|}{ Bcl-2 expression } & \multirow[t]{2}{*}{$p$} \\
\hline & High $n(\%)$ & Low n (\%) & & High $n(\%)$ & Low $n(\%)$ & \\
\hline ESCC & $35(34.31)$ & 67 (65.69) & $<0.01$ & 55 (53.92) & 47 (46.08) & $<0.01$ \\
\hline Adjacent tissues & 59 (57.84) & 43 (42.16) & & 33 (32.35) & 69 (67.65) & \\
\hline
\end{tabular}

ESCC: esophageal squamous cell carcinoma; $\chi^{2}$ test was applied, $\mathrm{p}<0.05$ was considered to be statistically significant.

serum were added to the upper chamber, while $600 \mu \mathrm{L}$ of DMEM medium with $10 \%$ FBS was placed in the bottom wells. After $24 \mathrm{~h}$ incubation at $37^{\circ} \mathrm{C}$, cells that had not moved to the lower chamber were removed. Subsequently, Crystal violet was used to stain the cells for $15 \mathrm{~min}$ before they were washed with PBS. Finally, five 200 optical fields were taken to count the number of invading cells. The average number of cells was calculated: data represent the mean \pm standard deviation.

\section{Clinical Outcome Assessment}

OS was defined as survival from the date of surgery to the date of death due to cancer. The OS of patients with ESCC was acquired in 102 cases: the mean OS was $40.60 \pm 2.11$ months (median: 31 months; range: $18-72$ months).

\section{Statistical Analysis}

Data were statistically analyzed using SPSS 18.0 software (SPSS Inc., Chicago, IL, United States). Pearson's $\chi^{2}$ test was applied to assess the association between Beclin-1 expression and Bcl-2 expression and several clinicopathological variables. The Kaplan-Meier method was used to determine the probability of survival, and the data were analyzed using a log-rank test. The Cox proportional hazards model was used for multivariate analysis of prognostic factors. Comparisons of quantitative data were analyzed using one-way ANOVA for multiple groups. $p$-values $<0.05$ were considered to be statistically significant.

\section{RESULTS}

\section{Beclin-1 and Bcl-2 Expression in ESCC}

The expression of Beclin-1 and Bcl-2 was evaluated in both tumor tissues and adjacent tissues using IHC. As shown in Table $134.31 \%(35 / 102)$ of ESCC tissues showed a high expression of Beclin-1. There was also a high expression rate of Beclin-1 in 57.84\% (59/102) of adjacent tissues, and this was significantly higher than of the expression in ESCC tissues $(p<0.01)$. In contrast, high expression of Bcl-2 was found in more ESCC tissues than in adjacent tissues $(53.92 \%$ vs. $32.35 \%$ respectively), and this difference was significant. Eventually, we detected that $34.31 \%$ (35/102) and 53.92\% (55/ 102) of the ESCC patients showed high expression of Beclin-1 and Bcl-2, respectively. High expression of both Beclin-1 and Bcl-2 was observed in 20 ESCC cases (Figure 1). Furthermore, the expression of Bcl-2 and Beclin-1 in tumor tissues was not correlated (Spearman's correlation: $p>0.05, r=0.047$; Table 2).

\section{Correlation of Beclin-1 and Bcl-2 Expression With Clinicopathological Features of ESCC}

The relationships between both Beclin-1 and Bcl-2 expression and the clinicopathological features of ESCC patients were analyzed. As shown in Table 3, ESCC with low expression of Beclin-1 was correlated with more advanced stages and lymph node metastasis. In addition, ESCC with high expression of Bcl-2 was correlated with deeper tumor invasion, more advanced

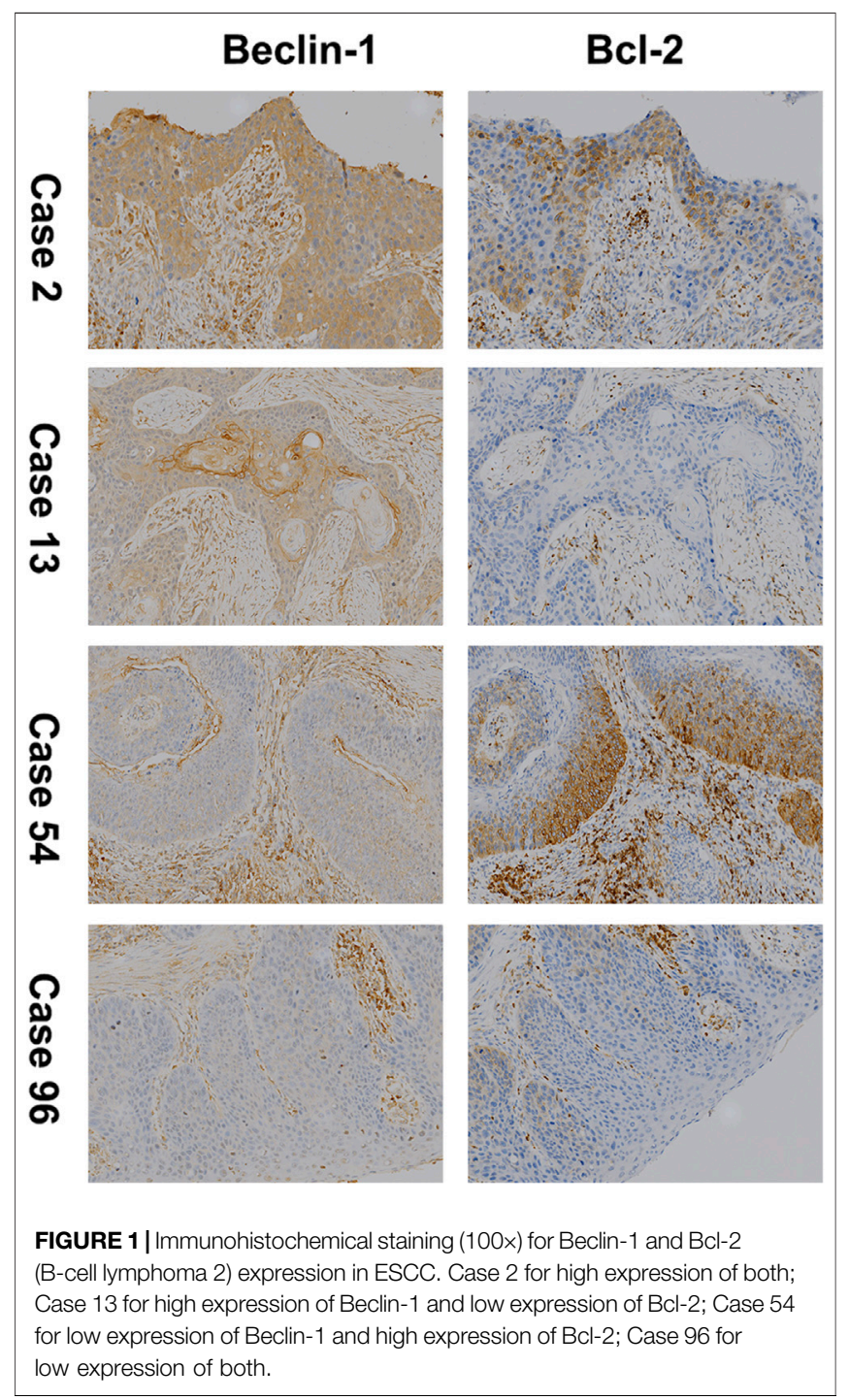


TABLE 2 | Association between Beclin-1 and Bcl-2 expression in ESCC.

Beclin-1

\begin{tabular}{ccccc}
\multicolumn{3}{c}{ Bcl-2 } & $p$ & $\boldsymbol{r}$ \\
\cline { 1 - 2 } High expression & Low expression & & \\
20 & 15 & 0.641 & 0.047 \\
35 & 50 & & \\
\hline
\end{tabular}

High expression

Low expression
ESCC: esophageal squamous cell carcinoma; $\mathrm{p}<0.05$ was considered to be statistically significant. The relationship between Beclin-1 and Bcl-2 was tested by Pearson's correlation analysis.

stages, and lymph node metastasis. However, the expression of both Beclin-1 and Bcl-2 in ESCC was not associated with age, gender, tumor size, or tumor differentiation.

\section{Correlation Between OS and the Changes in Both Beclin-1 and Bcl-2}

The 3- and 5-years OS of all patients involved was $46.08 \%$ and $26.47 \%$, respectively. In patients with high Beclin-1 expression, the 3 - and 5-years OS was $62.86 \%$ and $42.85 \%$, respectively; in contrast, in patients with low Beclin-1 expression, the 3- and 5years OS was $37.31 \%$ and $17.91 \%$, respectively (Figure 2A). On the other hand, in patients with high Bcl-2 expression, the 3- and 5 -years OS was $30.91 \%$ and $21.82 \%$, respectively, whereas, in patients with low Bcl-2 expression, the 3- and 5-years OS was $63.83 \%$ and $31.91 \%$, respectively (Figure $2 \mathbf{B}$ ).

\section{Univariate Analysis and Multivariate Analysis of OS in ESCC Patients}

Based on univariate analysis, a more advanced stage $(p<0.01)$, lymph node metastasis $(p<0.01)$, and low Beclin- 1 expression $(p<0.01)$ were identified as prognostic factors correlated with poor OS. Further multivariate analysis with these parameters in a Cox regression model confirmed that tumor stage (HR: 18.145; 95\% CI: 7.532-43.709; $p<0.01$ ), lymph node metastasis (HR: 18.586; 95\% CI: 7.021-49.204; $p<0.01$ ), and Beclin-1 expression (HR: 2.133; 95\% CI: 1.196-3.802; $p<0.01$; Table 4) were independent prognostic factors associated with OS. We also analyzed the correlation of the combination of Beclin-1 and Bcl-2 expression levels with OS. Cases in which ESCC patients had high expression of both Beclin-1 and Bcl-2 showed significantly longer survival than those with low expression of Beclin-1 (high expression of both Beclin-1 and Bcl-2 group vs. low expression of Beclin-1 and high expression of Bcl-2 group; $52.44 \pm 4.72$ vs. $27.92 \pm 2.44$; $p<0.01$; Figure 2C). When Bcl-2 was in a low-expression state, there was no survival difference in ESCC patients regardless of their expression levels of Beclin-1 (high expression of Beclin-1 and low expression of Bcl-2 group vs. low expression of both Beclin-1 and Bcl-2 group; $46.82 \pm 4.75$ vs. $44.80 \pm 3.86 ; p>$ 0.05; Figure 2D).

\section{Differential expression of Beclin-1 and Bcl-2 influences the invasiveness of the EC9706 ESCC cell line}

We established EC9706 cells with differential expression levels of Beclin-1 and Bcl-2 by pcDNA3.1-Beclin-1 or Bcl-2 plasmid and siRNA interference. After validation by western blotting (Figure 3), we examined how the different expression level of Beclin-1 and Bcl-2 influence invasiveness. EC9706 cells transfected with pcDNA3.1-Beclin-1 and pcDNA3.1-Bcl-2 showed a significant decrease in the number of invasive cells

\begin{tabular}{|c|c|c|c|c|c|c|c|c|c|}
\hline \multirow[t]{2}{*}{ Pathologic parameter } & \multirow[t]{2}{*}{ Case $n$} & \multicolumn{2}{|c|}{ Beclin-1 expression } & \multirow[t]{2}{*}{$\chi^{2}$} & \multirow[t]{2}{*}{$p$-value } & \multicolumn{2}{|c|}{ Bcl-2 expression } & \multirow[t]{2}{*}{$\chi^{2}$} & \multirow{2}{*}{$p$-value } \\
\hline & & High $n(\%)$ & Low n (\%) & & & High $n(\%)$ & Low $n(\%)$ & & \\
\hline Gender & & & & 0.54 & $>0.05$ & & & 0.15 & $>0.05$ \\
\hline Male & 65 & $24(36.92)$ & 41 (63.08) & & & $36(55.38)$ & $29(44.62)$ & & \\
\hline Female & 37 & $11(29.73)$ & $26(70.27)$ & & & 19 (51.35) & $18(48.65)$ & & \\
\hline Age & & & & 1.07 & $>0.05$ & & & 1.07 & $>0.05$ \\
\hline$\leq 60$ & 33 & $9(27.27)$ & 24 (72.73) & & & $14(42.42)$ & $19(57.58)$ & & \\
\hline$>60$ & 69 & $26(37.68)$ & 43 (62.32) & & & $41(59.42)$ & $28(40.58)$ & & \\
\hline Tumour diameter & & & & 0.37 & $>0.05$ & & & 2.6 & $>0.05$ \\
\hline$<3 \mathrm{~cm}$ & 57 & $21(36.84)$ & 36 (63.16) & & & $34(59.65)$ & $23(40.35)$ & & \\
\hline$\geq 3 \mathrm{~cm}$ & 45 & $14(31.11)$ & 31 (68.89) & & & $21(46.67)$ & $24(53.33)$ & & \\
\hline Differentiation & & & & 3.5 & $>0.05$ & & & 1.83 & $>0.05$ \\
\hline Well and moderate & 60 & $25(41.67)$ & 35 (58.33) & & & $29(48.33)$ & $31(51.67)$ & & \\
\hline Poor & 42 & $10(23.81)$ & 32 (76.19) & & & $26(61.90)$ & $16(38.10)$ & & \\
\hline Pathological stage & & & & 6.18 & $<0.05$ & & & 7.28 & $<0.01$ \\
\hline $1-\|$ & 44 & $21(47.73)$ & $23(57.27)$ & & & 17 (38.64) & 27 (61.36) & & \\
\hline III-IV & 58 & $14(24.14)$ & $44(75.86)$ & & & 38 (65.52) & $20(34.48)$ & & \\
\hline Lymph node & & & & 7.32 & $<0.01$ & & & 7.17 & $<0.01$ \\
\hline Positive & 70 & $18(25.71)$ & 52 (74.29) & & & $44(62.86)$ & $26(37.14)$ & & \\
\hline Negative & 32 & $17(53.13)$ & 15 (46.87) & & & 11 (34.38) & 21 (65.62) & & \\
\hline Depth of invasion & & & & 0.86 & $>0.05$ & & & 5.37 & $<0.05$ \\
\hline $\mathrm{T} 1, \mathrm{~T} 2$ & 46 & $18(39.13)$ & $28(60.87)$ & & & $19(41.30)$ & 27 (58.70) & & \\
\hline $\mathrm{T} 3, \mathrm{~T} 4$ & 56 & $17(30.36)$ & 39 (69.64) & & & $36(64.29)$ & $20(35.71)$ & & \\
\hline
\end{tabular}

$\mathrm{P}<0.05$ was considered statistically significant. $\chi^{2}$ test for categorical variables. 

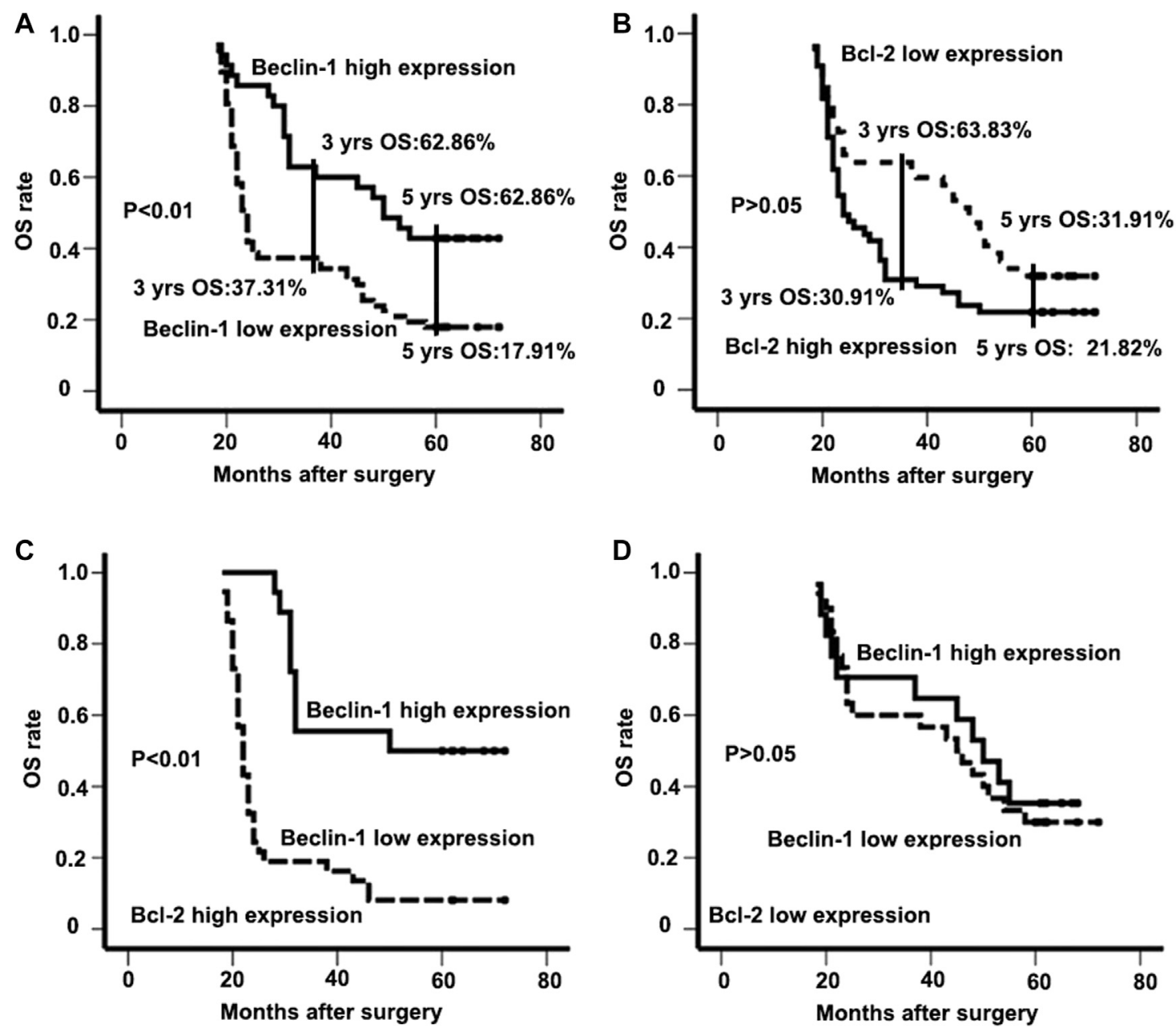

FIGURE 2| The expression patterns of Beclin-1 and Bcl-2 (B-cell lymphoma 2) in survival analysis of ESCC patients. (A) Kaplan-Meier analysis of overall survival for Beclin-1 expression in the whole study population; (B) overall survival for Bcl-2 (B-cell lymphoma 2) expression in the whole study population; (C) overall survival for the population with high-Bcl-2 (B-cell lymphoma 2) expression; (D) overall survival for the population with low- Bcl-2 (B-cell lymphoma 2) expression.

crossing the polycarbonate membrane of the Transwell invasion chamber $(p<0.01)$. In contrast, EC9706 cells transfected with pcDNA3.1-Bcl-2 and siBeclin-1 showed a significant increase in the number of invasive cells $(p<0.01$; Table 5). However, the effect of Beclin-1 expression levels on tumor cell invasiveness only appeared in combination with high Bcl-2 expression (there were no observed differences in other groups).

\section{DISCUSSION}

As one of the major malignant cancers, esophageal cancer caused about 500,000 deaths worldwide in 2018 [1]. In particular, China has a high incidence of ESCC and a high mortality rate, accounting for about half of all deaths worldwide. Importantly, the incidence of ESCC is increasing, so the disease poses a huge threat to public health and the economy [14-16]. As an important tumor suppressor gene, the study of Beclin-1, which has been identified as a novel Bcl-2-interacting protein, is vital to understanding the role of autophagy in tumorigenesis. In the present study, we investigated the interaction between Bcl-2 and Beclin-1 in ESCC. We found that Beclin-1 expression was associated with tumor stage and lymph node metastasis, and the expression of Beclin-1 in ESCC tissues was significantly lower than that in adjacent tissues, which suggests that autophagy activity was inhibited in ESCC. On the other hand, Bcl-2 expression in ESCC tissues was significantly higher than that in adjacent tissues. These results indicate that Beclin-1 plays an important role in the progression of ESCC and the autophagy defects that occur during more malignant cases of ESCC. Previous research showed that Beclin-1 contains a $\mathrm{BH} 3$ domain, which could bind to the $\mathrm{BH} 3$ receptor domain of $\mathrm{Bcl}-$ 2 and further inhibit the Beclin-1-induced autophagy [17]. Our results also suggest that Bcl-2 could bind to Beclin-1 through its receptor domain, which could inhibit autophagy activity and increase the malignancy of ESCC.

Autophagy plays a dual role in protecting and killing tumor cells [18]. Acting as strictly regulated biological processes, 
TABLE 4 | Univariate and multivariate analyses of factors for the prediction of overall survival.

\begin{tabular}{|c|c|c|c|c|}
\hline Variable & $\begin{array}{l}\text { Univariate analysis HR } \\
(95 \% \mathrm{Cl})\end{array}$ & $p$-value & $\begin{array}{l}\text { Multivariate analysis HR } \\
\qquad(95 \% \mathrm{Cl})\end{array}$ & $p$-value \\
\hline $\begin{array}{l}\text { Gender } \\
\text { Male } \\
\text { female }\end{array}$ & 1.503 (0.881-2.563) & 0.134 & & \\
\hline $\begin{array}{l}\text { Age } \\
\quad \leq 60 \\
>60\end{array}$ & $0.852(0.483-1.505)$ & 0.582 & & \\
\hline $\begin{array}{l}\text { Tumor diameter } \\
\qquad 3 \mathrm{~cm} \\
\geq 3 \mathrm{~cm}\end{array}$ & $0.815(0.491-1.354)$ & 0.430 & & \\
\hline $\begin{array}{l}\text { Differentiation } \\
\text { Well and moderate } \\
\text { Poor }\end{array}$ & $1.068(0.656-1.738)$ & 0.792 & & \\
\hline $\begin{array}{l}\text { Pathologic stage } \\
\qquad \text { I, II } \\
\text { III, III }\end{array}$ & $12.944(4.811-34.826)$ & $<0.01$ & 18.145 (7.532-43.709) & $<0.01$ \\
\hline $\begin{array}{l}\text { Lymph node } \\
\text { Positive } \\
\text { Negative }\end{array}$ & 31.281 (10.487-93.305) & $<0.01$ & 18.586 (7.021-49.204) & $<0.01$ \\
\hline $\begin{array}{l}\text { Invade depth } \\
\text { T1, T2 } \\
\text { T3, T4 }\end{array}$ & $2.048(0.996-4.213)$ & 0.051 & & \\
\hline $\begin{array}{l}\text { Beclin-1 } \\
\text { High } \\
\text { Low }\end{array}$ & 1.859 (1.020-3.388) & $<0.05$ & 2.133 (1.196-3.802) & $<0.05$ \\
\hline $\begin{array}{l}\text { Bcl-2 } \\
\text { High } \\
\text { Low }\end{array}$ & 1.363 (0.779-2.384) & 0.278 & & \\
\hline
\end{tabular}

autophagy and apoptosis also play an important role in maintaining cell homeostasis [19]. The relationship between autophagy and apoptosis has become a prominent area of research in cancer science. In our study, we explored the important interaction between the autophagy-specific protein Beclin-1 and the anti-apoptotic protein Bcl-2, which are potential targets for autophagy and apoptosis. Therefore, we investigated the combined role of apoptosis and autophagy in the prognosis of ESCC. Immunohistochemical results revealed that the 3- and 5-years OS rates in patients with low Beclin-1 expression were significantly lower than in patients with high Beclin-1 expression. In addition, our analyses indicated that Beclin-1 was an independent predictor of OS in ESCC patients with high Bcl-2 expression. Thus, Beclin-1 should be analyzed in combination with $\mathrm{Bcl}-2$ expression to assess the prognosis of ESCC patients.

In further analysis, we established cells with different Beclin-1 and Bcl-2 expression states using plasmid vectors and siRNA before making in vitro observations of the invasiveness of tumor cells. We found that the expression of Beclin-1 significantly affected the number of invasive cells in EC9706 only when Bcl-2 was highly expressed. One of our previous study [20] and another study by Farkas et al. [21] showed that Beclin-1 and Bcl-2 could be prognositc predictive marks in ESCC respectively. Our present study indicate that increased expression of Beclin-1 protein may reduce the invasiveness of EC9706 cells only in combination with high Bcl-2 expression levels. Thus, Beclin-1 must be used as an independent prognostic factor for ESCC patients in combination with Bcl-2 expression. On the other hand, our study also raises the following question: why were different Beclin-1 expression levels not relative to invasiveness and prognosis in ESCC patients with low Bcl-2 expression? Previous research has shown that apoptosis could eliminate tumor cells $[22,23]$. In addition, autophagy is known to play a role in protecting tumor cell survival in tumorigenesis and progression with intact apoptosis $[24,25]$. Furthermore, autophagic cell death may only occur when apoptosis is inhibited $[23,26]$. Our results suggest that a relationship between autophagy and apoptosis may also exist in ESCC. Of course, we cannot eliminate other complex molecular mechanisms and signaling pathways as potential regulators in the occurrence and progression of ESCC. However, the present study's limitation is that it is a retrospective analysis of a comparatively small sample, other studies and potential mechanisms must therefore be evaluated in future research studies.

In summary, we have shown that autophagy-specific protein expression and autophagy activity were inhibited in ESCC. Differences in autophagy and apoptotic states and their activities may promote malignant tumor differentiation, which in turn may lead to a more aggressive esophageal squamous cell phenotype and a worse survival prognosis. Importantly, Beclin-1 is a promising prognostic biomarker and therapeutic target for patients with ESCC in the highBcl-2-expression population. 


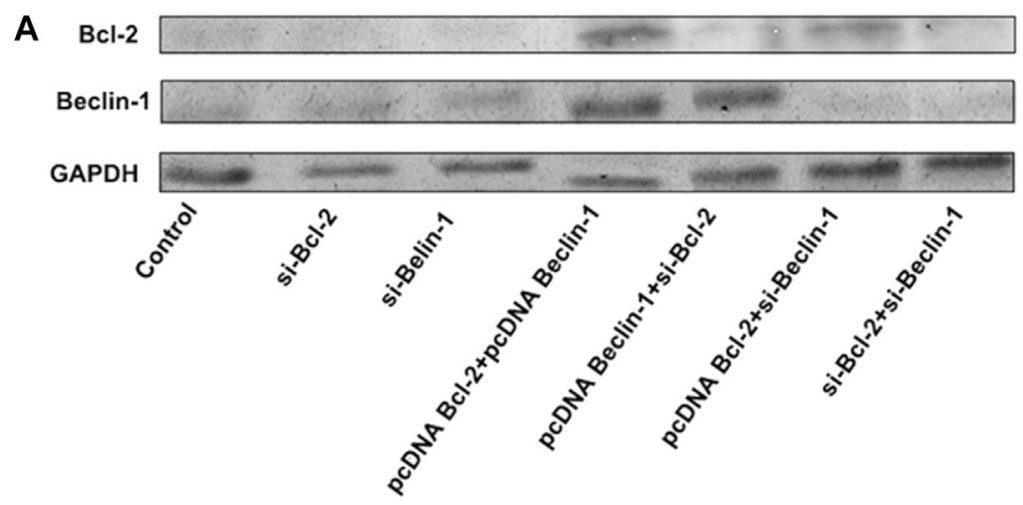

B
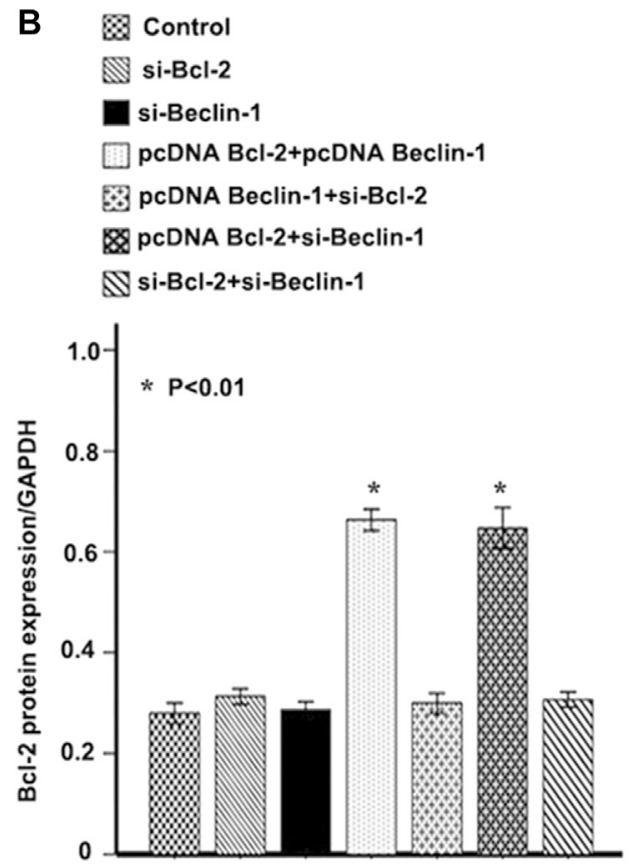

C $\otimes$ Control

$\mathbb{Q}$ si-Bcl-2

si-Beclin-1

D pcDNA Bcl-2+pcDNA Beclin-1

[5.) pcDNA Beclin-1+si-Bcl-2

pcDNA Bcl-2+si-Beclin-1

Si-Bcl-2+si-Beclin-1

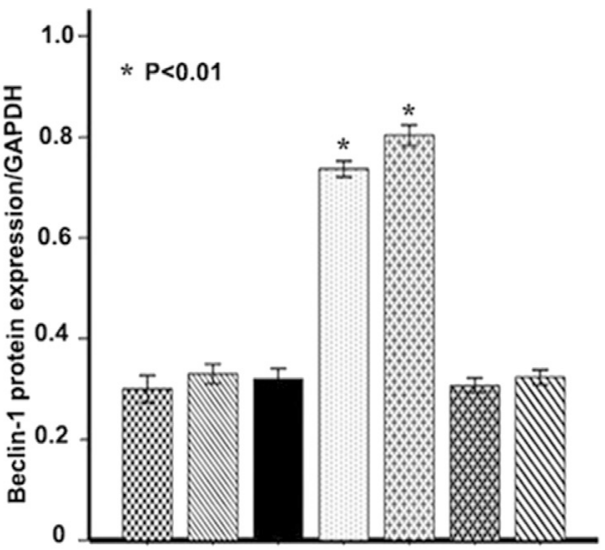

FIGURE 3 | Confirmation of Bcl-2 (B-cell lymphoma 2) and Beclin-1 overexpression or knockdown. (A) Western blot analysis of Bcl-2 (B-cell lymphoma 2) and Beclin-1 expression in EC9706 cells transfected with pcDNA3.1-Beclin-1 or Bcl-2 (B-cell lymphoma 2) plasmid and siRNA interference. (B) Relative Bcl-2 (B-cell lymphoma 2) protein expression/GAPDH (Glyceraldehyde-3-phosphate dehydrogenase) in each group. (C) Relative Beclin-1 protein expression/GAPDH (Glyceraldehyde-3-phosphate dehydrogenase) in each group. ${ }^{\star} p<0.01$ vs. control group, calculated with one-way ANOVA analysis of variance.

TABLE 5 | The comparison of invasiveness of EC9706 cells in relation to expression of Beclin-1 and Bcl-2.

Groups

Bcl-2 expression ${ }^{\mathrm{a}, \%}$
Beclin-1 expression $^{a}, \%$

$30.00 \pm 2.65$

$33.00 \pm 2.00$

$32.00 \pm 2.00$

$73.67 \pm 1.53$

$80.33 \pm 2.08$

$30.67 \pm 1.53$

$32.33 \pm 1.53$
Number

of invasive cells

$243.32 \pm 38.23$

$225.36 \pm 28.16$

$235.36 \pm 31.26$

$52.65 \pm 12.24^{*}$

$240.46 \pm 32.18$

$381.97 \pm 32.31^{\star}$

$242.12 \pm 36.23$

si-Bcl-2 + si-Beclin-1

$30.67 \pm 1.53$

${ }^{a}$ Mean \pm standard deviation, relative to $\mathrm{GAPDH} ;{ }^{*} \mathrm{p}<0.01$ vs. untransfected control group, calculated with one-way ANOVA analysis of variance. 


\section{DATA AVAILABILITY STATEMENT}

The original contributions presented in the study are included in the article/Supplementary Material, further inquiries can be directed to the corresponding author.

\section{ETHICS STATEMENT}

The studies involving human participants were reviewed and approved by the Clinical Ethics Committee of Ruijin Hospital.

The patients/participants provided their written informed consent to participate in this study.

\section{AUTHOR CONTRIBUTIONS}

HD undertook the collection of clinical-pathological data and patient follow-up. FL conducted the immunochemistry experiments. MS conducted in vitro cell experiments. HD, JC, and LZ performed the statistical analysis. HL and $\mathrm{JH}$ made contributions to conception and design. All authors were

\section{REFERENCES}

1. Bray F, Ferlay J, Soerjomataram I, Siegel RL, Torre LA, Jemal A Global cancer statistics 2018: GLOBOCAN estimates of incidence and mortality worldwide for 36 cancers in 185 countries. CA: A Cancer J Clin (2018). 68(6):394-424. doi:10. $3322 /$ caac. 21492

2. Siegel RL, Miller KD, Jemal A Cancer statistics, 2016. CA: A Cancer J Clin (2016). 66(1):7-30. doi:10.3322/caac.21332

3. Torre LA, Siegel RL, Ward EM, Jemal A Global cancer incidence and mortality rates and trends-an update. Cancer Epidemiol Biomarkers Prev (2016). 25(1): 16-27. doi:10.1158/1055-9965.epi-15-0578

4. Yu L, Chen Y, Tooze SA Autophagy pathway: cellular and molecular mechanisms. Autophagy (2018). 14(2):207-15. doi:10.1080/15548627.2017. 1378838

5. Saha S, Panigrahi DP, Patil S, Bhutia SK Autophagy in health and disease: a comprehensive review. Biomed Pharmacother (2018). 104:485-95. doi:10.1016/ j.biopha.2018.05.007

6. Li Q, Liu Y, Sun M Autophagy and alzheimer's disease. Cell Mol Neurobiol (2017). 37(3):377-88. doi:10.1007/s10571-016-0386-8

7. Mialet-Perez J, Vindis C Autophagy in health and disease: focus on the cardiovascular system. Essays Biochem (2017). 61(6):721-32. doi:10.1042/ ebc20170022

8. Hall TM, Tétreault M-P, Hamilton KE, Whelan KA Autophagy as a cytoprotective mechanism in esophageal squamous cell carcinoma. Curr Opin Pharmacol (2018). 41:12-9. doi:10.1016/j.coph.2018.04.003

9. Hamurcu Z, Delibaşı N, Geçene S, Şener EF, Dönmez-Altuntaş H, Özkul Y, et al. Targeting LC3 and Beclin-1 autophagy genes suppresses proliferation, survival, migration and invasion by inhibition of Cyclin-D1 and uPAR/Integrin $\beta 1 /$ Src signaling in triple negative breast cancer cells. J Cancer Res Clin Oncol (2018). 144(3):415-30. doi:10.1007/s00432-017-2557-5

10. Huang F, Wang B-R, Wang Y-G Role of autophagy in tumorigenesis, metastasis, targeted therapy and drug resistance of hepatocellular carcinoma. World J Gastroenterol (2018). 24(41):4643-51. doi:10.3748/wjg. v24.i41.4643

11. Shen H, Yin L, Deng G, Guo C, Han Y, Li Y, et al. Knockdown of Beclin-1 impairs epithelial-mesenchymal transition of colon cancer cells. J Cel Biochem (2018). 119(8):7022-31. doi:10.1002/jcb.26912 involved in the writing of the manuscript and approved the final version.

\section{FUNDING}

This study was supported by the Shanghai Municipal Commission of Health and Family Planning Outstanding Academic Leaders Training Program (2017BR055).

\section{CONFLICT OF INTEREST}

The authors declare that the research was conducted in the absence of any commercial or financial relationships that could be construed as a potential conflict of interest.

\section{ACKNOWLEDGMENTS}

The authors would like to acknowledge Dr. Ruyuan Zhang and Dr. Qin Ye for their assistance on this manuscript.

12. Sohn EJ, Park HT Natural agents mediated autophagic signal networks in cancer. Cancer Cel Int (2017). 17:110. doi:10.1186/ s12935-017-0486-7

13. Zhang XF, Yang Y, Zhang J, Cao W Microvesicle-containing miRNA-153-3p induces the apoptosis of proximal tubular epithelial cells and participates in renal interstitial fibrosis. Eur Rev Med Pharmacol Sci (2019). 23(22):10065-71. doi:10.26355/eurrev_201911_19574

14. Zeng H, Zheng R, Zhang S, Zuo T, Xia C, Zou X, et al. Esophageal cancer statistics in China, 2011: estimates based on 177 cancer registries. Thorac Cancer (2016). 7(2):232-7. doi:10.1111/1759-7714.12322

15. Malhotra GK, Yanala U, Ravipati A, Follet M, Vijayakumar M, Are C Global trends in esophageal cancer. J Surg Oncol (2017). 115(5):564-79. doi:10.1002/jso.24592

16. Pakzad R, Mohammadian-Hafshejani A, Khosravi B, Soltani S, Pakzad I, Mohammadian $M$, et al. The incidence and mortality of esophageal cancer and their relationship to development in Asia. Ann Transl Med (2016). 4(2):29. doi:10.3978/j.issn.23055839.2016.01.11

17. Xu H-D, Qin Z-H Beclin 1, bcl-2 and autophagy. Adv Exp Med Biol (2019). 1206:109-26. doi:10.1007/978-981-15-0602-4_5

18. Feng F, Zhang M, Yang C, Heng X, Wu X The dual roles of autophagy in gliomagenesis and clinical therapy strategies based on autophagic regulation mechanisms. Biomed Pharmacother (2019). 120:109441. doi:10.1016/j.biopha. 2019.109441

19. Kasprowska-Liśkiewicz D The cell on the edge of life and death: crosstalk between autophagy and apoptosis. Postepy Hig Med Dosw (Online) (2017). 71(0):825-41. doi:10.5604/01.3001.0010.4672

20. Du H, Che J, Shi M, Zhu L, Hang JB, Chen Z, et al. Beclin 1 expression is associated with the occurrence and development of esophageal squamous cell carcinoma. Oncol Lett (2017). 14(6):6823-8. doi:10. 3892/ol.2017.7015

21. Farkas R, Pozsgai E, Bellyei S, Cseke L, Szigeti A, Vereczkei A, et al. Correlation between tumor-associated proteins and response to neoadjuvant treatment in patients with advanced squamous-cell esophageal cancer. Anticancer Res (2011). 31(5):1769-75.

22. Badrinath N, Yoo SY Mitochondria in cancer: in the aspects of tumorigenesis and targeted therapy. Carcinogenesis (2018). 39(12): 1419-30. doi:10.1093/carcin/bgy148 
23. Hasanain M, Sahai R, Pandey $\mathrm{P}$, et al. Microtubule disrupting agent-mediated inhibition of cancer cell growth is associated with blockade of autophagic flux and simultaneous induction of apoptosis. Cell Prolif (2020). 53(4):e12749. doi:10.1111/cpr.12749

24. Geng X, Ren Y, Wang F, Tian D, Yao X, Zhang Y, et al. Harmines inhibit cancer cell growth through coordinated activation of apoptosis and inhibition of autophagy. Biochem Biophysical Res Commun (2018). 498(1):99-104. doi:10.1016/j.bbrc.2018.02.205

25. Athamneh K, Alneyadi A, Alsamri H, Alrashedi A, Palakott A, El-Tarabily KA, et al. Origanum majorana essential oil triggers p38 MAPK-mediated protective autophagy, apoptosis, and caspase-dependent cleavage of P70S6K in colorectal cancer cells. Biomolecules (2020). 10(3):412. doi:10.3390/biom10030412
26. Law BYK, Michelangeli F, Qu YQ, Xu SW, Han Y, Mok SWF, et al. Neferine induces autophagy-dependent cell death in apoptosis-resistant cancers via ryanodine receptor and $\mathrm{Ca}(2+)$-dependent mechanism. Sci Rep (2019). 9(1): 20034. doi:10.1038/s41598-019-56675-6

Copyright (c) $2021 \mathrm{Du}, \mathrm{Luo}, \mathrm{Shi}$, Che, Zhu, Li and Hang. This is an open-access article distributed under the terms of the Creative Commons Attribution License (CC BY).

The use, distribution or reproduction in other forums is permitted, provided the original author(s) and the copyright owner(s) are credited and that the original publication in this journal is cited, in accordance with accepted academic practice. No use, distribution or reproduction is permitted which does not comply with these terms. 